brennt, während sich metallisches Wismuth in der Retorte ausscheidet. Fährt man fort zu erhilzen, so steigl das Thermometer über $160^{\circ}$, bis dann plölzlich unter heftiger Explosion der Apparat zertrümmert wird.

In chemischer Beziehung verhält sich das Wismuthälhyl ganz wie ein organisches Radical. Selzt man zu der weingeistigen Lösung desselben eine Lösung von Brom oder Jod, so verschwindet deren Farbe unter bedeutender Wärmeentwickelung und Bildung von Jod - oder Brombismäthyl. Jedoch sind die Verbindungen weniger beständig als die enlsprechenden des Stibâthyls. Lälst man z. B. eine weingeistge Lösung von Jodbismäthyl eine kurze Zeit stehen, so scheidet sich Jodwismuth aus. Das Wismuthäthyl löst sich leicht in verdünnter Salpetersäure und die Lösung enthält jedenfalls salpetersaures Bismäthyloxyd. Dampft man hingegen die salpetersaure Lösung ab, so bleibt reines salpetersaures Wismulhoxyd zurück. Die Beschreibung der Verbindungen des Wismuthäthyls soll einer zweiten Abhandlung vorbehallen bleiben.

Ueber die unorganischen Bestandtheile des Lycopodium chamaecyparissus und Lyc. clavatum und insbesondere deren Thonerdegehalt;

von Dr. A. Aderholdt.

Die gegen den Thonerdegehalt der Pflanzen erhobenen Zweifel, welche weder der Fürst zu Salın-Horstmar durch seine Untersuchung des Lyc. complanalum (Journ. pr. Ch. v. Erdmann und Marchand XXXVIII, 347), worin er 38 pC. Thonerde land, noch Willstein, welcher in fast allen Gartensträuchern Thonerde angab (Rep. d. Pharm. XLIV, 332), zu 
beseitigen vermochten, indem ihnen der Vorwurf gemacht wird, dafs sie keine genügende Garantie dafür gäben, ob das untersuchte Material wirklich von anhängenden Bodentheilchen frei gewesen sey (cf. Jahresb. v. Liebig und Kopp 1847-48, S. 1097), veranlafsten den Verfasser, zur endlichen Entscheidung dieser Frage sorgfältige Untersuchungen anzustellen.

Da es nicht unwahrscheinlich schien, dafs die Pflanze, welche der Fürst zu Sal m - Hors $t$ m a r als Lycopodium complanatum anführt, dieselbe sey, als die neuerlich als Lyc. chamaecyparissus beschriebene, so wurde zunächst die letztere, und als sich in ihr ein bedeutender Thonerdegelıalt zeigte, auch das Lyc. clavatum untersucht, welches ebenfalls als reich an Thonerde befunden wurde. Der Thonerdegehalt beider Lycopodien war so bedeutend, dafs sich der Verf. veranlafst fühlt, den drei Abtheilungen, in welche man die Pflanzen nach ihren vorwiegenden Aschenbeslandtheilen eingetheilt hat, nämlich Kali -, Kalk - und Kieselpflanzen, noch eine vierte, die der Thonerdeptlanzen, hinzuzufügen.

Da nun auch in Betreff der übrigen Aschenbestandtheile die genannten Lykopodien keine sehr gewöhnliche Zusammensetzung zeigen, so folgt hier das Ergebnils der angestellten Analysen, bei deren Berechnung die Aequivalentgewichtszahlen zu Grunde gelegt sind, die in Liebig und Kopp's Jahresbericht für 1850 angenommen sind.

\section{Lycopodium chamaecyparissus.}

Es wurde Anfangs März 1851 gesammelt. Der Standort desselben war der Venusberg bei Bonn, eine tertiäre, Braunkohlenlager haltende Gebirgsmasse. Die Pflanze war auf dem Gipfel dieses Berges auf einem freien, vom Walde unkränzten Platze gewachsen; in gröfserer Entfernung davon befinden sich Alaunhiulten. 
Der Wasserauszug des Bodens, auf welchem dic Pflanze wuchs, reagirte schwach sauer, enthielt weder Salpetersüure noch Phosphorsäure, noch Chlor, wohl aber Schwefelsäure, und zwar auch Spuren freier Schwefelsäure, wie aus dem Schwarzwerden einer Probe des Wasserauszugs bei vorsichtigem Eindampfen mit Zucker geschlossen wurde, und in ammoniakalischen Auszuge wurde Ulminsäure aufgefunden.

Es wurden, wie das auch von Salı-Horstmar geschehen war, nur die grünen Theile der Pflanze der Analyse unterworfen, indem das Kraul elwas über dem Boden abgeschnitten wurde. Das so erhaltene Material, welches am Tage vor dem Einsammeln vom Regen abgespült war, erwies sich als so sauber, dafs an den meisten Exemplaren selbst mil Hülfe der Loupe kein Staub bemerkt werden konnte. Nichtsdestoweniger wurde es sorglältigem Waschen unterworfen, bis die geringen Spuren von Verunreinigung vollkommen verschwunden waren. Da es darauf ankam, Material zu erhalten, welches von allen anhängenden Bodentheilchen frei war, so bedurfle es dieser Behandlung, selbst auf die Gefahr hin, einen Verlust an löslichen Salzen zu erleiden, welcher jedoch nicht bedeulend seyn kann, da der Schnillfächen nur wenige waren, und dic äufsere Rinde der Pflanze ein Ausgezogenwerden verhindert.

Die so gereinigle Pflanze wurde verkohit, mil Wasser ausgezogen und darauf cingeäschert. Der Wasscrauszug reagirte schwach alkalisch. Er wurde mil Salpetersäure angesäuest und zur Fällung des Chlors mit salpetersaurem Silberoxyd verselzt, wobei indefs nur eine Trübung entstand, wefshalb das Chlor in einem besonderen Auszuge der Kohle mil verduinnter Salpelersăure bestimmt werden mufste. Jener Wasserauszug wurde nach der Abscheidung des Silbers durch Schwefelwasserstoff eingedampft und mil der Asche vereinigt.

Die ganze Masse wurde nun mit Salzsäure auf dem Wasserbade zur Trockne verdampft. Im löslichen Theile wurde die 


\section{Aderholdt, über die unorganischen Bestandtheile}

Hauptmenge der Thonerde durch Fällen mit Ammoniak und Ausziehen mil Kalilauge bestimmt; es zeigte sich aber, dals cinestheils nicht die ganze Menge der Thonerde aus dem Ammoniakniederschlage durch Kali ausgezogen werden konnte, sondern slets noch eine beträchtliche Menge bei der Fällung des Eisens mit niederfiel, anderntheils aus dem in Salzsäure unlöslichen Theile noch eine Quantilät Thonerde durch Schmelzen mil saurem schwefelsaurem Kali gewonnen wurde. Hierdurch wurde der Thonerdegehalt noch um cin Belrächtliches vermehrt.

Die Phosphorsäure, welche mit der Thonerde niederfiel, wurde durch Glühen des Niederschlags mit Kieselsäure und Soda von jener geschieden und aus der Lösung als phosphorsaure Ammoniakmagnesia gewonnen.

Die Magnesia wurde von den Alkalien durch Quecksilberoxyd getrennt.

Das frische Kraut enthiclt 50 pC. Wasser, das bei $100^{\circ}$ gelrocknele 6,1 pC. Asche.

Nach zwei Analysen ergab sich folgende Zusammenselzung :

\begin{tabular}{|c|c|c|c|c|}
\hline $\mathrm{Al}^{2} \mathrm{O}^{3}$ & . & $\begin{array}{c}\text { In } 1,223 \mathrm{Gr} . \\
0,614\end{array}$ & $\begin{array}{r}\text { Focentische zusa } \\
\text { nach Abug des } \\
\mathbf{5 1 , 8 5}\end{array}$ & $\begin{array}{l}\text { smensetzum } \\
\text { pC. }\end{array}$ \\
\hline $\mathrm{SiO}^{3}$. & . & $\mathbf{0 , 1 6 1}$ & 13,60 & 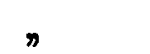 \\
\hline KO . & . & 0,147 & 12,42 & 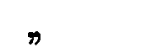 \\
\hline $\mathrm{CaO}$. & . & 0,064 & 5,41 & $n$ \\
\hline $\mathrm{SO}^{\mathrm{s}}$ & . & 0,052 & 4,38 & $n$ \\
\hline $\mathrm{MgO}$. & . & 0,047 & 3,97 & $\eta$ \\
\hline $\mathrm{PO}^{5}$ & . . & 0,043 & 3,63 & $\pi$ \\
\hline $\mathrm{Mn}^{3} \mathrm{O}^{4}$ & . & 0,026 & 2,20 & n \\
\hline $\mathrm{Fe}^{2} \mathrm{O}^{3}$ & . . & 0,009 & 0,76 & $\pi$ \\
\hline $\mathrm{NaO}$. & . & 0,009 & 0,76 & $\pi$ \\
\hline $\mathrm{NaCl}$. & . & $\frac{0,012}{1,184}$ & 1,01 & 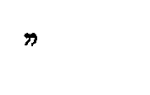 \\
\hline Verlust & . & $\begin{array}{r}0,039 \\
1,223 .\end{array}$ & & \\
\hline
\end{tabular}


des Lycopodium chamaecyparissus u. Lyc. clavatum. 115

$$
\begin{aligned}
& \text { Sauerstoffmenge der Oxyde MO }=6,3 \quad 30,5 \\
& \begin{array}{ll}
\eta \quad \text { Thonerde }=24,2 \\
\Rightarrow \text { Säuren }=11,8 \\
\hline 42,3 .
\end{array}
\end{aligned}
$$

Eine ganz ähnliche Zusammensetzung zeigte auch im November desselben Jahres gesanımeltes grünes Lycopodium, welches noch keine Sporen getragen halte. Der Standort war derselbe, wie bei dem im März gesammellen.

Aschenmenge des bei $100^{\circ}$ getrocknelen Kraules $4,5 \mathrm{pC}$.

In 100 Theilen

In $3,770 \mathrm{Gr}$.

$\mathrm{Al}^{2} \mathrm{O}^{3}$. $\quad 2,146$

$\mathrm{SiO}^{3}$. $\quad 0,485$

KO . . . 0,441

$\mathrm{CaO}$. . 0,180

$\mathrm{SO}^{3}$. . 0,121

$\mathrm{MgO} \cdot \quad \cdot 0,120$

$\mathrm{PO}^{5}$. . . 0,104

$\mathrm{Mn}^{3} \mathrm{O}^{4}$. . $\quad 0,075$

$\mathrm{Fe}^{2} \mathrm{O}^{3}$. . 0,028

$\mathrm{NaO}$. . . 0,005

$\mathrm{NaCl} \cdot \frac{0,036}{3,741}$

Verlust . $\frac{0,029}{3,770 \text {. }}$ nach Abzug des Verlustes

57,364 pC.

12,964 ,

11,788

4,812 ,

3,234 ,

3,208 ,

2,708

2,005 ,

0,748 ,

0,134 ,

0,962 ,

In dieser lelzlen Analyse wurde indefs das Chlor nicht bestimmt, sondern wegen der Analogie dieser Analyse mil der vorigen zu 0,022 Gr. auf 3,770 Gr. Asche angenommen.

Das Natron wurde hier nach Abscheidung des Kalis als Kaliumplatinchlorid aus dem zersetzten Nalriumplatinclslorid als schwefelsaures Salz beslimmt.

Von Kupfer, welches John im Lyc. complan. angiebt, zeigte sich keine Spur; ebensowenig von Brom und Jod, wohl aber von Tưtansāure. 
Um diese lelztere nachzuweisen, wurde eine grofse Menge Asche mil Wasser und dann mil Salzsäure ausgezogen, wodurch kein Titan gewonnen wude. Der Rückstand wurde mit Salzsäure zur 'Trockne verdampft und nach dem Schmelzen mit saurem schwefelsaurem Kali mit kaltem Wasser behandelt. Aus dem Filtrate schieden sich beim Sieden Flocken ab, die sich durch die violelte Färbung der Phosphorsalzperle in der Reductionsflamme als Tilan zu crkennen gaben.

Diese Aufnahme von Titan hat nichts Befremdendes, da sich in der Nähe des Standorles des untersuchten Iycopodiums titanhallige Eisensteine finden.

\section{Lycopodium clavalum.}

Von dieser Pflanze wurden ebenfalls nur die grünen Theile untersucht. Das Material wurde in März auf dem Venusberge bei Bonn gesammelt und sehr sorgfälligem Waschen unlerworfen. Da indefs bei diesem Lycopodium der Fall eintreten kann, dafs anhängende, in den Blatlwinkeln verhorgene Bodentheilchen sich der Reinigung entzietien, so wurden zur qualitativen Ermitlelung der Thonerde von einer gewissen Quantität die Bläller abgestreifl, und da bei dieser Manipulation auch die Rinde grölstentheils mit absprang, so gelang es, sehr reines Material zu erhalten. Die Untersuchung dieses so vorgerichteten Materials ergab bedeutende Mengen Thonerde.

Hierauf wurden die sauber gewaschenen und genau besichtigten Pflanzen (welche von den Blältern nicht befreil waren) eingeäschert, was schr leicht und schnell von Slatlen ging.

Auch hier mufsten noch nicht unbedeutende Mengen von 'Thonerde von Kieselsäure und Eisenoxyd getrennt und der durch Kali aus dem Ammoniakniederschlage ausgezogenen Hauptportion zugerechnet werden. Zwei Analysen führten zu folgenden Resultaten :

Aschenmenge des bei $100^{\circ}$ getrocknelen Krautes 4,7 pC. 
des Lycopodium chamaecyparissus $u$. Lyc. clavatum. 117

\begin{tabular}{|c|c|c|c|c|}
\hline$\left.A\right|^{2} \mathrm{O}^{3}$ & . . & $\begin{array}{c}\text { In } 1,402 \mathrm{Gr} . \\
0,348\end{array}$ & $\begin{array}{r}\text { In } 100 \text { Theiler } \\
\text { der Kohlensă } \\
\text { Verlust } \\
26,65\end{array}$ & $\begin{array}{l}\text { nach Abzug } \\
\text { ure und des } \\
\text { es } \\
\text { pC. }\end{array}$ \\
\hline KO & . . & 0,316 & 24,19 & $\eta$ \\
\hline $\mathrm{SiO}^{3}$ & . . & 0,182 & 13,94 & » \\
\hline $\mathrm{CaO}$. & . . & 0,104 & 7,96 & $»$ \\
\hline $\mathrm{MgO}$ & . . & 0,085 & 6,51 & $\eta$ \\
\hline $\mathrm{PO}^{\mathbf{5}}$ & . . & 0,070 & 5,36 & $»$ \\
\hline $\mathrm{SO}^{3}$ & . . & 0,064 & 4,90 & $n$ \\
\hline $\mathrm{Na} \mathrm{Cl}$. & - . & 0,043 & 3,29 & $»$ \\
\hline $\mathrm{Mn}^{3} \mathrm{O}^{4}$ & . . & 0,033 & 2,53 & » \\
\hline $\mathrm{K} \mathrm{Cl}$ & . . & 0,031 & 2,37 & $»$ \\
\hline $\mathrm{Fe}^{2} \mathrm{O}^{3}$ & . & 0,030 & 2,30 & n \\
\hline $\mathrm{CO}^{2}$ & . & $\begin{array}{r}0,043 \\
1,349\end{array}$ & - & \\
\hline
\end{tabular}

Verlust $\cdot \frac{0,053}{1,402}$

$\begin{aligned} \text { Sauerstoffmenge der Oxyde MO } & =10,5 \\ & \text { Thonerde }=12,4\end{aligned} \quad 22,9$

Die Vergleichung der Aschen beider Lycopodien zeigh interessanle Verhältnisse. Es sind defshalb die Resultate hier noch einmal zusammengestellt :

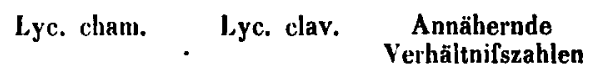

\begin{tabular}{|c|c|c|c|c|c|c|c|}
\hline $\mathrm{Al}^{2} \mathbf{O}^{3}$ & . & . & . & ${ }^{\circ}$ & 51,85 & 26,65 & $2: 1$ \\
\hline $\mathrm{SiO}^{3}$ & - & • & $\theta^{\prime}$ & . & 13,60 & 13,94 & $1: 1$ \\
\hline KO & . & . & . & . & 12,42 & 24,19 & $1: 2$ \\
\hline $\mathrm{CaO}$. & . & . & . & . & 5,41 & 7,96 & $3: 4$ \\
\hline $\mathrm{SO}^{3}$ & • & . & . & . & 4,38 & 4,90 & $1: 1$ \\
\hline $\mathrm{PO}^{\mathrm{s}}$ & . & . & . & . & 3,63 & 5,36 & $2: 3$ \\
\hline $\mathrm{MgO}$. & . & . & . & . & 3,97 & 6,51 & $1: 2$ \\
\hline
\end{tabular}


118 Aderholdt, über die unorganischen Bestandtheile elc.

$$
\text { Lyc. cham. Lyc. clav. }
$$

\begin{tabular}{|c|c|c|}
\hline $\mathrm{Mn}^{3} \mathrm{O}^{4}$ & & . \\
\hline $\mathrm{NaO}$ & . & . \\
\hline$e^{2} 0^{3}$ & & \\
\hline
\end{tabular}

$\mathrm{NaCl} \mathrm{Cl} . \quad . \quad 1,01$

$\mathrm{K} \mathrm{Cl} \mathrm{.} \mathrm{.} \mathrm{.} \mathrm{.} \mathrm{.} \mathrm{-}$

0 der Basen . . $\quad 30,5$

0 der Säuren . . $\quad 11,8$

0 der Oxyde MO

0 der Thonerde.
2,53

2,30

3,29

2,37

22,9

13,3

10,5

12,4
Annähernde Verhälttifszahlen

$1: 1$

$1: 3$

$1: 3$

$5: 4$

$1: 1$

$1: 2$

$2: 1$.

Es ergaben sich also folgende Verhältnisse :

$\begin{array}{lcccc} & \begin{array}{c}\text { Oder Oxyde } \\ \text { M0 }\end{array} & \begin{array}{c}0 \text { der } \\ \text { Säuren }\end{array} & \begin{array}{c}\text { O der } \\ \text { Thonerde }\end{array} & \begin{array}{c}\text { Annähernde } \\ \text { Verhältnifszahien }\end{array} \\ \text { Lyc. cham. } & 6,3 & 11,8 & 24,2 & 1: 2: 4 \\ \text { Lyc. clav. } & 10,5 & 13,3 & 12,4 & 2: 2: 2 .\end{array}$

Andere auf demselben Boden gewachsene Pflanzen, wie Eiche, Ficlite und Birke, zeigten keine Spur von Thonerde.

Es ist somit nachgewiesen, dafs die Thonerde wirklich von den Pflanzen aufgenommen werden kann, und dafs sie in jenen Lycopodien den wesentlichsten Bestandtheil ausmacht, durch welche dic Klasse der Thonerdepflanzen eröflnet wird.

Es werde hier noch angeführt, dafs in dem Behufs der organischen Bestandheile des Lyc. chamaecyp. dargestellten Wasserauszuge dieser Pflanze nicht unbeträchtliche Mengen von Thonerde aufgefunden wurden.

Was schliefslich die vom Fürsten zu Salm-Horstmar beobachtete saure Reaction der Wurzelfasern von Lyc. complanatum belriff, so bemerklen wir dieselbe auch bei dem Lyc. chamaccyparissus.

Die vorstehenden Analysen sind im Laboralorium des Hrn. Dr. Bödeker, Privaldocenten in Bonn, und unter dessen gütiger Leitung ausgefühtt. 
Fuchs, uiber die Löslichkeit und den Fydratzustand etc. 119

Ueber die wäberen Details vergl. Dissert. de part. anory. Lycopodii chamaecyp. et clavati, Bonnae 1852.

\title{
Ueber die Löslichkeit und den Hydratzustand der Kieselsäure ;
}

\author{
von J. Fuchs,
}

Assistenten am chemischen Laboratoritm des Prof. Fresenius zu Wiesbaden.

Unter der Ueberschrift : „Ueber die Eigenschaften der Kieselsäure", findet sich in dem Journal für praclische Chemie von Erdmann und Marchand (Band XLII, Jahr 1847) eine Abhandlung von Leonh. Doveri (Ann. de Chim. et de Phys. T. XXI, p. 40), in welcher derselbe über die Löslichkeit des Kieselsäurchydrates und dessen Zusammensetzung einige Mittheilungen macht, welche mit den Resultaten der nachfolgend angestellten Versuche nicht in Einklang zu bringen sind, und gleichzeitig die Formel Si $\mathrm{O}_{3}$ für die Kieselsäure bedingen, während nach der von inir gefundenen Zusammensetzung der Kieselsäurehydrate die Formel $\mathrm{Si}_{2}$ fast allein für zulässig gehalten werden kann.

Das zu den nachfolgendell Versuchen verwendele Kiesclsäurehydrat wurde durch Einleiten von Kieselfluorgas in Wasser dargestelll- und so lange ausgewaschen, bis die ablaufende Flüssigkeit durchaus keine Reaction auf Kieselfluorwasserstoffsäure mehr zeigte.

1. Löslichkeit des Kieselsäurehydrates in Wasser und Säuren.

Das feuchte, gallertarlige Kieselsäurehydrat wurde mehrmal - und zwar ein und derselbe 'Theil - mit Wasser und sodann mit Chlorwasserstoffsäure kalt und kochend digerirl, durch doppeltes Filter filtrirt und das Filtrat in einer gewogenen Platinschale zur 